\section{Anterior transposition vs anterior and nasal transposition of inferior oblique muscle in treatment of dissociated vertical deviation associated with inferior oblique overaction}

MF Farid ${ }^{1,2}$
'Department of

Ophthalmology, Benha Faculty of Medicine, Benha University, Benha, Egypt

${ }^{2}$ Department of Ophthalmology, Benha University Hospital, Benha, Egypt

Correspondence: MF Farid, Department of Ophthalmology, Benha University Hospital, 11 Al Ekhlas Street, New Benha, Benha 13512, Egypt Tel: +20 1003354636 E-mail: Mohamed_fathy_ 10@yahoo.com

Received: 17 April 2015 Accepted in revised form: 2 November 2015 Published online: 8 January 2016

\begin{abstract}
Purpose To compare results of two surgical techniques; anteriorization (ATIO) vs anterior nasalization (ANT) of IO muscle in management of DVD associated with IOOA. Methods Twenty-one patients with DVD associated with IOOA were included in this study. Group A consists of $\mathbf{1 1}$ patients who underwent ATIO and group B with 10 patients who underwent anterior transposition of IO to the nasal border of inferior rectus ANT. All patients were followed for at least 6 months postoperatively. The primary outcome variables were changes in DVD in primary position and side gazes, IO action and $\mathrm{V}$ pattern.

Results The average of correction of DVD in primary position, in adduction and in abduction was 10.63 PD, 24.6 PD $(P<0.001)$ and 0.45 PD5 $(P>0.05)$ in ATIO group and 14.6 PD, 25.2 PD and 1.7 PD $(P<0.001)$ in ANT group respectively. Mean IOOA decreased from $+2.0 \pm 0.7$ to $+0.18 \pm 0.4$ in group $\mathrm{A}(P<0.001)$ and from $+2.5 \pm 0.7$ to $+0.1 \pm 0.5(P<0.001)$ in group $B$. Mean $V$ pattern was corrected from 19.18 $\pm 7.1 \mathrm{PD}$ to $11.18 \pm 4.9 \mathrm{PD}(P<0.01)$ in group $\mathrm{A}$ and from $17.8 \pm 7.9 \mathrm{PD}$ to $6.0 \pm 2.49 \mathrm{PD}(P<0.001)$ in group $B$. In group $B$, two patients developed hypotropia of 2 and 4 PD and one patient developed consecutive exotropia.

Conclusions In DVD associated with IOOA, both surgical techniques are almost similar in alleviating true hypertropia in side gaze, IOOA, and V pattern. ANT gives more
\end{abstract}

statistically significant DVD correction in primary position and in abduction while in adduction; there is no significant difference between both groups. However, ANT may induce hypotropia and consecutive horizontal strabismus.

Eye (2016) 30, 522-528; doi:10.1038/eye.2015.257; published online 8 January 2016

\section{Introduction}

Dissociated vertical deviation DVD is a condition characterized by spontaneous upward drifting of one or both eyes when binocularity was blocked. ${ }^{1}$ In some cases, this drift is associated with excyclotorsion, latent Nystagmus and head tilt. When fixation is regained, the up-drifted eye will return slowly to the primary position without any accompanying re-corrective movement in the contralateral eye, so the term dissociated arise. ${ }^{1}$

Many surgical procedures have been tried for treatment of DVD, For example, superior rectus recession with or without Faden, inferior rectus resection and recession of inferior oblique. Anterior transposition of the inferior oblique insertion at the temporal border of inferior rectus insertion (inferior oblique anteriorization, ATIO) is the current most agreed form of treatment. ${ }^{2}$ Stager et al proposed transposition of the inferior oblique muscle nasal to the nasal border of the inferior rectus muscle insertion. ${ }^{3}$ The purpose of this study was to compare the efficacy of Stager's technique to standard ATIO for the treatment of DVD with concurrent inferior oblique overaction IOOA. 


\section{Patients and methods}

This prospective uncontrolled study complied with the Declaration of Helsinki and was approved by the ethics committee of Benha Faculty of Medicine, Benha University. Patients of the second group were informed about traditional surgical techniques, which have a relatively well-known rate of success, and given alternatives to treatment before enrolling in this study. Written informed consent was obtained from all the patients after full explanation of both procedures. This study includes patients with either manifest or latent DVD of $\geq 12$ PD in primary position in one or both eyes associated with IOOA. Inclusion decision was not affected by concurrent or previously done horizontal muscle surgery. Patients who underwent previous oblique or vertical rectus muscle surgery were excluded. DVD patients with normal IO action were also excluded in addition to those associated with paralytic or craniofacial anomalies. High level of cooperation was needed for optimum pre- and post-operative measurements so uncooperative patients were also excluded. Pre-operative and post-operative measurements were undertaken by one assistant who was masked to the procedure that was performed. IO action was assessed and graded on a scale of -4 to +4 . DVD in primary position was measured by alternate base-down prism cover test while patient fixing a $6 \mathrm{~m}$ distant accommodative target and wearing his/her refractive correction. ${ }^{4}$

True hypertropia or hypotropia was measured in side gaze with base-down prism in front of the adducted eye. The amount of base-down prism needed to neutralize any hypotropic shift of the abducted eye represented true hypertropia or hypotropia. Any additional base-down prism needed to neutralize the hyperdeviation of the adducted eye represented DVD of the adducted eye. ${ }^{5}$ $\mathrm{V}$ pattern was measured as the difference between horizontal deviations in up and down gazes.

Patients were randomized to receive anteriorization of inferior oblique at the lateral border of inferior rectus muscle insertion, ATIO (group A) or anterior and nasal transposition of inferior oblique at the nasal border of inferior rectus muscle insertion, ANT (group B).

Postoperatively, DVD in primary position, in adduction and in abduction was measured. Total hyperdeviation in adduction, inferior oblique action, concurrent true hyperor hypotropias, V pattern, and complications were also recorded.

All surgeries were done by the author (MFF). Inferior temporal fornix conjunctival incision was used to gain access to the inferior oblique. A muscle hook was used to isolate lateral and inferior rectus muscles. After hooking and isolation of inferior oblique, muscle insertion was disinserted from the sclera by cutting it in between two hemostats placed as close to insertion as possible. In group A, after cauterizing the cut ends, locking, double armed 6-0 vicryl sutures were passed through the stump. Muscle was then re-inserted immediately temporal to inferior rectus insertion. ${ }^{6}$ In group B, muscle was attached to the sclera at nasal border of inferior rectus insertion by using 5-0 Mersiline (Ethicon) sutures. Non-absorbable sutures were used in the second group to avoid retraction and slippage of new muscle insertion as recommended by Stager et al. ${ }^{3}$ It is important firstly to attach posterior fibers of inferior oblique $1 \mathrm{~mm}$ nasal to the nasal border of inferior rectus tendon then to attach the anterior fibers $3 \mathrm{~mm}$ nasal to the first suture, so the insertion was not fanned and was $\sim 2-3 \mathrm{~mm}$ in width. In both groups, the inferior oblique muscle was then inspected to ensure that there are no remaining posterior fibers that had not been anteriorly transposed. The conjunctiva was then closed with interrupted 6-0 vicryl sutures. Unilateral surgery was done for unilateral cases and bilateral surgery was done symmetrically in bilateral cases even if DVD and/or IOOA were asymmetrical. Post-operative measurements were recorded at 1,3 , and 6 months. At last postoperative visit, DVD of 0-4 PD was considered an excellent result, 5-9 PD a good result and 10-14 PD a fair result. These data were tabulated and analyzed using the computer program SPSS (IBM Corp, Armonk, NY, USA). Statistical significance was tested using paired $t$-test and willcoxon test with $P$-value $<0.05$ was considered statistically significant.

\section{Results}

A total of 21 DVD patients associated with IOOA met the inclusion criteria and were divided into two groups; group A which contains 11 patients who underwent inferior oblique anteriorization (ATIO) and group B which involves 10 patients who underwent anterior and nasal transposition of inferior oblique (ANT). Two patients, one in each group had unilateral DVD associated with an ipsilateral IOOA and they received a unilateral procedure. The rest of patients in both groups received bilateral surgery for bilateral DVD and IOOA.

Mean follow-up was $8.3 \pm 2.4$ months for group A and $9.5 \pm 3.6$ months for group B. Male to female ratio was 1:3.75 in group A and 1:4 in group B. The mean age at the time of surgery for group A was $12 \pm 4.07$ years (range, 7-20) and for group B was 13.6 \pm 5.03 (range, 7-22).

In group A, ATIO was done isolated in four patients, combined with horizontal muscle surgery in four patients, and on top of previously operated horizontal strabismus for infantile esotropia in three patients. All patients who underwent combined ATIO and horizontal muscle surgery were esotropic. They underwent ATIO combined with bimedial rectus recession (average $4.5 \pm 0.4 \mathrm{~mm}$ ), 
which resulted in improvement of their esotropia from $30 \pm 4.08$ PD preoperatively to $3.5 \pm 3.4$ PD (Table 1).

In group B, ANT was done isolated in three patients, with horizontal surgery in four patients and three patients had previous horizontal muscle surgery for infantile esotropia. In patients who underwent combined ANT and horizontal muscle surgery, three patients were esotropic with an average pre-operative deviation of 31.6 \pm 5.7 PD and one patient had 40 PD exotropia. ANT combined with bimedial recession (average $4.6 \pm 0.5 \mathrm{~mm}$ ) were done to esotropic patients, which resulted in an average post-operative deviation of $2 \pm 2 \mathrm{PD}$ ET in two cases with development of consecutive XT of 20 PD in the third case that was later corrected by bimedial rectus advancement with a final deviation of 8 PD XT. The exotropic patient underwent combined ANT with $8 \mathrm{~mm}$ bilateral lateral rectus recession that resulted in $8 \mathrm{PD}$ XT postoperatively. Preoperatively, two patients, one in each group had no fusion with worth's four dot test. All other patients had different grades of stereopsis 'ranging from 40 to 800 arcsec $^{\prime}$.

All patients in both groups had preoperative inferior oblique overaction. Mean pre - and post-operative IOOA in both groups are shown in Table 1. In both groups, improvement of IOOA was statistically significant $(P<0.001)$ although difference between both groups was statistically insignificant $(P \geq 0.05)$. Persistent IOOA $(+1)$ was observed postoperatively in two eyes in each group. In ANT group, two patients, with one eye in each, developed post-operative IO underaction (-1).

In ATIO group before the operation, DVD in primary position averaged 19.9 $\pm 4.6 \mathrm{PD}(12-25 \mathrm{PD}), 28.45 \pm 5.3 \mathrm{PD}$ (20-35 PD) in adduction, and 2.6 \pm 1.02 PD (1-4 PD) in abduction. Mean true hyperdeviation of left eye in adduction (in right gaze) was $8.9 \pm 5.2 \mathrm{PD}$ and for the right eye in left gaze was $5.9 \pm 3.7 \mathrm{PD}$. After the operation, DVD averaged 9.27 \pm 3.79 PD (4-14 PD) in primary position $(P<0.001), 3.8 \pm 2.04 \mathrm{PD}(0-6 \mathrm{PD})$ in adduction $(P<0.001)$, and $2.18 \pm 1.07$ PD $(0-4$ PD) in abduction $(P>0.05)$. These figures represent an average $53.4 \%$ reduction in primary position, $86.6 \%$ reduction in adduction, and $16.1 \%$ reduction in abduction (Table 1 ). The true hypertropia in adduction was corrected to $1.9 \pm 2.1 \mathrm{PD},(P<0.001)$ in the left eye and $1.4 \mathrm{D} \pm 3.6 \mathrm{PD}$ $(P \leq 0.01)$ in the right eye (Table 1$)$.

Before the operation In ANT group, DVD averaged $18.5 \pm 4.4 \mathrm{PD}(12-25 \mathrm{PD})$ in primary position, $28.8 \pm 5.5$ PD (20-36 PD) in adduction, and 2.7 \pm 0.9 PD (1-4 PD) in abduction. Mean true hyperdeviation in adduction was $9.4 \pm 4.8 \mathrm{PD}$ in left eye and $6.9 \pm 5.7 \mathrm{PD}$ in right eye. After the operation, DVD averaged $3.9 \pm 1.19 \mathrm{PD}(2-6 \mathrm{PD})$ in primary position $(P<0.001), 3.6 \pm 1.4 \mathrm{PD}(2-6 \mathrm{PD})$ in adduction $(P<0.001)$, and $1.0 \pm 0.8 \mathrm{PD}(0-2 \mathrm{PD})$ in abduction $(P<0.001)$. These figures represent an average

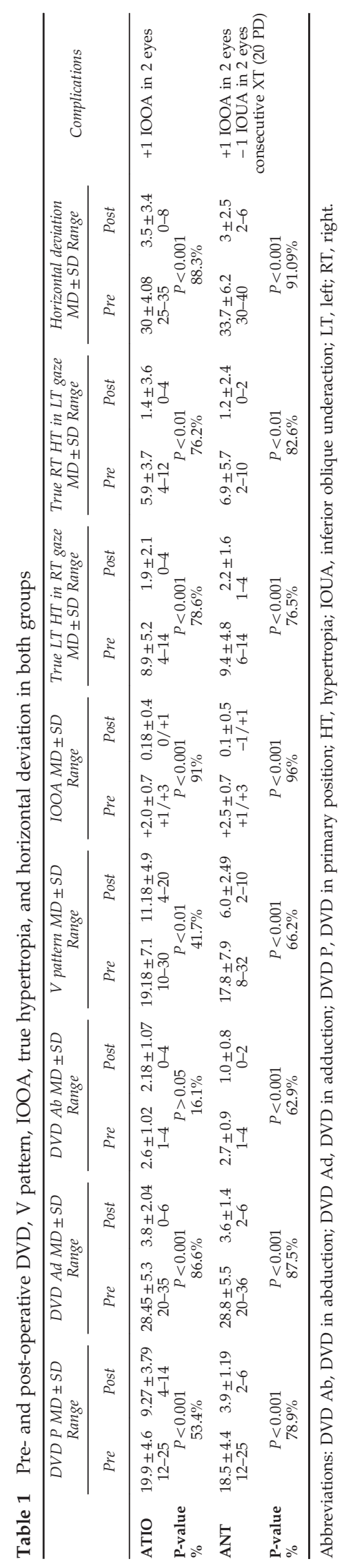


$78.9 \%$ reduction in primary position, $87.5 \%$ reduction in adduction, and $62.9 \%$ reduction in abduction (Table 1 ). The true hypertropia in adduction was corrected to $2.2 \pm 1.6 \mathrm{PD},(P<0.001)$ in the left eye, and $1.2 \pm 2.4 \mathrm{PD}$ $(P=<0.01)$ in the right eye (Table 1$)$.

The average of correction of DVD in primary position, in adduction and in abduction was $10.63 \pm 2.1 \mathrm{PD}$, $24.6 \pm 3.9 \mathrm{PD}$, and $0.45 \pm 0.5 \mathrm{PD}$ in ATIO group and 14.6 $\pm 3.4 \mathrm{PD}, 25.2 \pm 4.4 \mathrm{PD}$, and $1.7 \pm 0.4 \mathrm{PD}$ in ANT group, respectively (Figure 1). Overall, ANT gives more correction in primary position and in abduction, which is statistically significant $(P \leq 0.05)$ while in adduction, there is insignificant difference in correction of DVD among both groups $(P>0.05)$.

Mean correction of $\mathrm{V}$ pattern is $8 \pm 2.6 \mathrm{PD}$ in group $\mathrm{A}$ $(P \leq 0.01)$ and $11.8 \pm 5.7 \mathrm{PD}$ in group $\mathrm{B}(P<0.001)$. These figures represent an average $41.7 \%$ reduction in ATIO group and $66.2 \%$ reduction in ANT group (Table 1). The difference between both groups was statistically insignificant $(P \geq 0.05)$. (Figure 1$)$.

In both groups, $100 \%$ of eyes achieved excellent post-operative result (DVD 0-4 PD) in abduction. In adduction, $63 \%$ of ATIO group achieved that result compared with $80 \%$ of ANT group. In primary position, only $10 \%$ of ATIO group achieved excellent result compared with 70\% of ANT group (Figure 2, Table 2). Eighty-two percent of eyes before surgery had DVD $>14$ PD in primary position in group A. Among them, $44 \%$ had a good outcome and $56 \%$ had a fair outcome. For eyes with preoperative DVD $<14$ PD $(18 \%)$, $50 \%$ had an excellent outcome and $50 \%$ had a good outcome. In group B, all eyes (100\%) with preoperative
DVD $<14$ PD (20\% of eyes) achieved an excellent outcome. In eyes who had preoperative DVD > 14 PD $(80 \%), 37.5 \%$ had an excellent outcome and $62.5 \%$ had a good outcome.

Comitant hypertropia in primary position was observed before surgery in two patients in group $\mathrm{A}$ and three patients in group B (mean, group A; 3.5 PD, group B; 4 PD). All patients in both groups had a comitant hypertropia on adduction while none of patients in both groups had on abduction. Mild post-operative hypotropia (2 and $4 \mathrm{PD}$ ) was observed in primary position of two eyes in two patients in ANT group. These were the same eyes that develop $(-1)$ inferior oblique underaction postoperatively. No further interventions were required as hypotropia was mild and innocuous. One patient in group B, who was esotropic and underwent combined Bimedial recession with bilateral ANT, developed post-operative consecutive exotropia of $20 \mathrm{PD}$. This patient required additional intervention (medial rectus advancement) and finally resulted in 8 PD XT. In both groups, no patients developed defective elevation in abduction (antielevation syndrome).

\section{Discussion}

Several management strategies had been investigated thoroughly to control DVD. For instance, large superior rectus recession with or without Faden, inferior rectus muscle resection either isolated or combined with superior rectus muscle recession. ${ }^{7-10}$ Surgery on vertical recti was found to induce changes in lid position, vertical diplopia and limitation of upgaze. ${ }^{11}$ Weakening of

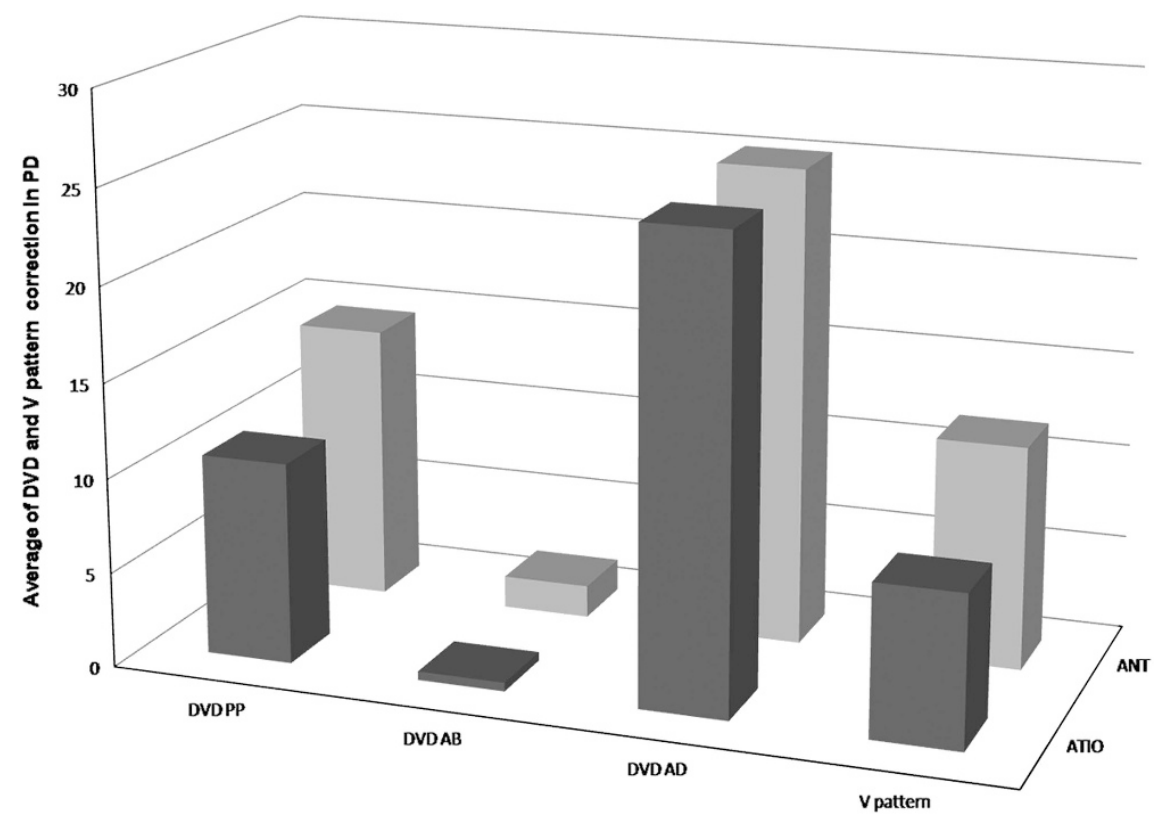

Figure 1 Mean correction of V pattern and DVD in different position of gaze. 


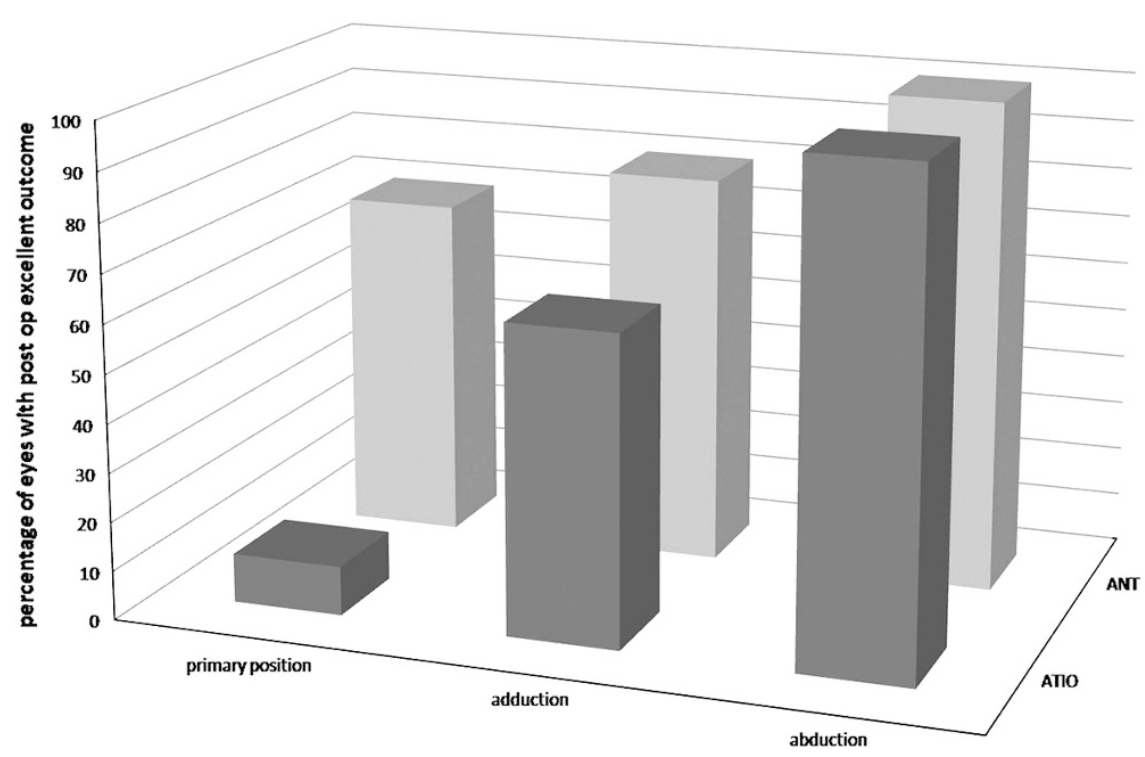

Figure 2 Percentage of post-operative excellent outcome (0-4 PD) in different position of gaze.

Table 2 Pre-operative and post-operative DVD: percentage of eyes within each alignment group in different gaze positions

\begin{tabular}{|c|c|c|c|c|c|c|}
\hline \multirow[t]{2}{*}{$D V D(P D)$} & \multicolumn{3}{|c|}{ Preoperative } & \multicolumn{3}{|c|}{ Postoperative } \\
\hline & Primary & Adduction & Abduction & Primary & Adduction & Abduction \\
\hline \multicolumn{7}{|l|}{ Group A } \\
\hline 0-4 & 0 & 0 & $100 \%$ & $10 \%$ & $63 \%$ & $100 \%$ \\
\hline $5-9$ & 0 & 0 & 0 & $35 \%$ & $37 \%$ & 0 \\
\hline $10-14$ & $18 \%$ & 0 & 0 & $55 \%$ & 0 & 0 \\
\hline$>14$ & $82 \%$ & $100 \%$ & 0 & 0 & 0 & 0 \\
\hline \multicolumn{7}{|l|}{ Group B } \\
\hline 0-4 & 0 & 0 & $100 \%$ & $70 \%$ & $80 \%$ & $100 \%$ \\
\hline 5-9 & 0 & 0 & 0 & $30 \%$ & $20 \%$ & 0 \\
\hline $10-14$ & $20 \%$ & 0 & 0 & 0 & 0 & 0 \\
\hline$>14$ & $80 \%$ & $100 \%$ & 0 & 0 & 0 & 0 \\
\hline
\end{tabular}

overacting Inferior oblique muscle has been proposed as the standard procedure for DVD associated with IOOA. ${ }^{11,12}$ IO myectomy and standard IO recession were found not effective compared with ATIO that remains the standard treatment for combined DVD and IOOA. ${ }^{13}$

Mims and Bremer claimed that ATIO changes IO action from an elevator to a depressor due to Lockwood's ligament effect. ${ }^{14,15}$ Stager proposed the role of neuro fibrovascular bundle that was supported with clinical, radiological, and histological evidences. ${ }^{16}$ Elliot and Nankin ${ }^{11}$ compared ATIO to IO recession in treating IOOA and concluded that ATIO changes the action of IO from an elevator to a depressor. Mims and Woods ${ }^{14}$ noted improvement in DVD and IOOA when evaluating bilateral ATIO in treatment of 61 IOOA patients. Burke et $a l^{12}$ found that ATIO was effective in controlling DVD in 19 out of 22 eyes. Black ${ }^{5}$ reported good results of ATIO in controlling cases with small DVD. Engman ${ }^{17}$ also reported excellent results of ATIO in 56\% of cases with small DVD and in $25 \%$ of cases with large DVD (>15 PD). In ATIO group of the current study, 50\% of patients with smaller amounts of DVD achieved excellent results, whereas none of patients with DVD > 14 PD achieved an excellent outcome. Milot et al ${ }^{18}$ concluded that ATIO is more effective in eliminating IOOA $(100 \%$ of cases) but the procedure is less predictable for DVD with $29.4 \%$ recurrences during a follow-up period of 28.2 months. Coats ${ }^{19}$ results also were less encouraging regarding ATIO in treatment of DVD. The current study finds out that ATIO is effective in controlling IOOA (91\% reduction). For DVD, ATIO was more effective in controlling DVD in adduction (86.6\% reduction) than in primary position and in abduction (53.4\% and $16.1 \%$, respectively).

Stager et $\mathrm{al}^{3}$ in 2001 proposed transposing IO not only anteriorly but also nasally to the nasal border of IR. In this 
technique, the IO is positioned anterior to $x$ axis and nasal to $y$ axis, which changes IO from an elevator and extorter to a depressor and intorter in adduction. Furthermore, in 2003, they studied the effect of this new procedure on IOOA in a diverse group of patients. ${ }^{20}$ Among them, two cases had DVD associated and ANT resulted in elimination of both. Ellis argued that ANT will lead to an Intorsion effect in upgaze. However, he agreed that ANT causes less extorsion in upgaze compared with ATIO. ${ }^{21}$ Fard $^{22}$ concluded that ANT was effective in controlling DVD, IOOA, and V pattern in his 10 patient's study with $53 \%$ of his patients with large DVD achieved an excellent outcome and $47 \%$ had a fair outcome. In ANT group of the current study, only $37.5 \%$ of patients with DVD > 14 PD achieved an excellent outcome and $62.5 \%$ had a good outcome. Our ANT results exactly match that of Fard study in small DVD patients where $100 \%$ of patients achieved an excellent outcome. V pattern was corrected by $59.5 \%$ in Fard study compared with $66.2 \%$ in ANT group of the current study. True hypertropia in side gaze was significantly corrected in Fard study and in the current series.

Stager et $a l^{20}$ reported limitation of elevation in some of his unilateral and bilateral ANT cases. IO underaction developed in 3 eyes out of 20 eyes in Fard study, whereas in our study, it developed in only 2 out of 19 eyes of ANT group. In these patients, post-operative hypotropia of 2 and 4 PD were also reported. Post-operative hypotropia was a reported complication of ANT procedure (4 cases) in Fard study and was also reported following ATIO in Black, Burke, Bremer and Quinn studies.5,12,15,23 One case in the current series developed consecutive exotropia of 20 PD. This patient had long-standing infantile ET associated with unilateral DVD and IOOA and the patient had no fusion on worth's four dot test. This patient was treated by bimedial rectus recession and unilateral ANT. Consecutive exotropia was treated by advancement of both medial recti 6 weeks after the primary surgery with a final outcome of 8 PD XT. Post-operative XT was a reported complication of ANT in Stager's study ${ }^{20}$ and he advocated that XT was due to extreme degree of nasalization" 4 to $5 \mathrm{~mm}$ " nasal to IR nasal border which will cause distortion of IR belly by the nasally shifted ancillary origin of IO. In our case, this was not the issue as the amount of nasalization was the standard amount done for all patients. Lack of preoperative fusion and/or a unilateral procedure might be the precipitating factor for such complication.

Despite its limitations, and up to our knowledge, this study is the first to compare the results of ATIO and ANT in management of DVD with IOOA. Generally, both procedures are almost equally effective in controlling IOOA, V pattern, and DVD in adduction. ANT gives significantly better results for DVD in primary position and in abduction. Both procedures are effective in controlling true hypertropia in side gaze with no significant difference in between. Persistent IOOA was reported nearly equally in both groups. In the current series, post-operative primary position hypotropia associated with IO underaction was an exclusive complication to ANT group in which also, a case of consecutive exotropia was reported.

In conclusion, compared with ATIO, ANT gives better control for DVD associated with IOOA especially in abduction and in primary position although this comes with the cost of higher rates of post-operative complications.

\section{Summary}

What was known before

- inferior oblique anterior transposition was the standard treatment of combined DVD and IOOA. Anterior nasal transposition of inferior oblique is relatively a new technique for treatment of combined DVD and IOOA.

What this study adds

- Comparison between both surgical techniques regarding correction of DVD in different positions of gaze, $\mathrm{V}$ pattern, IOOA as well as rate of post-operative complications.

\section{Conflict of interest}

The author declares no conflict of interest.

\section{References}

1 von Noorden GK, Campos EC. Binocular Vision and Ocular Motility. Mosby: St Louis, MO, USA, 2002, pp 378-385.

2 Esswein MB, von Noorden GK, Coburn A. Comparison of surgical methods in the treatment of dissociated vertical deviation. Am J Ophthalmol 1992; 113: 287-290.

3 Stager Sr DR, Beauchamp GR, Stager DR Jr. Anterior and nasal transposition of the inferior oblique muscle: A preliminary case report on a new procedure. Binocul Vis Strabismus Q 2001; 16: 43-44.

4 Mallette RA, Repka MX, Guyton DL. Superior rectus suspension recession for dissociated vertical deviation: a report of 59 operations. Binocular Vision 1987; 2: 209-215.

5 Black BC. Results of anterior transposition of the inferior oblique muscle in incomitant dissociated vertical deviation. J AAPOS 1997; 1: 83-87.

6 Kratz RE, Rogers GL, Bremer DL, Leguire LE. Anterior tendon displacement of the inferior oblique for DVD. J Pediatr Ophthalmol Strabismus 1989; 26: 212-217.

7 Schwartz T, Scott W. Unilateral superior rectus muscle recession for the treatment of dissociated vertical deviation. J Pediatr Ophthalmol Strabismus 1991; 28: 219-222.

8 Caputo A, Raab EL, Ruttum M. Management of dissociated vertical deviation. J Pediatr Ophthalmol Strabismus 1999; 36: 208-212. 
9 Jampolsky A. Management of vertical strabismus. Trans New Orleans Acad Ophthalmol 1986; 34: 141-171.

10 Bacal DA, Nelson LB. Anterior transposition of the inferior oblique muscle for both dissociated vertical deviation and/or inferior oblique overaction: results of 94 procedures in 55 patients. Binocul Vis Eye Muscle Surg Q 1992; 7: 219-225.

11 Elliott RL, Nankin SJ. Anterior transposition ofthe inferior oblique. J Pediatr Ophthalmol Strabismus 1981; 18: 35-38.

12 Burke JP, Scott WE, Kutshke PJ. Anterior transposition of the inferior oblique muscle for dissociated vertical deviation. Ophthalmology 1993; 100: 245-250.

13 Gonzalez C, Klein B. Myectomy and anterior transposition of the inferior oblique: a new surgical procedure and its results in 49 operations. Binoc Vis Eye Muscle Surgery Qtrly 1993; 8: 249-258.

14 Mims III JL, Wood RC. Bilateral anterior transposition of the inferior obliques. Arch Ophthalmol 1989; 107: 41-44.

15 Bremer DL, Rogers GL, Quick LD. Primary-position hypotropia after anterior transposition of the inferior oblique. Arch Ophthalmol 1986; 104: 229-232.

16 Stager DR. The neurofibrovascular bundle of the inferior oblique muscle as the ancillary origin of that muscle. J AAPOS 1997; 1: 216-225.

17 Engman JH, Egbert JE, Summers CG, Young TL. Efficacy of inferior oblique anterior transposition placement grading for dissociated vertical deviation. Ophthalmology 2001; 108: 2045-2050.

18 Milot JM, Tremblay C, Ouellette C. Anterior transposition of the inferior oblique for dissociated vertical deviation with inferior oblique overaction. Can J Ophthalmol 1994; 29: 284-287.

19 Coats DK, Paysse EA, Stager DR. Surgical management of $\mathrm{V}$-pattern strabismus and oblique dysfunction in craniofacial dysostosis. J AAPOS 2000; 4: 338-342.

20 Stager Jr DR, Beauchamp GR, Wright WW, Felius J, Stager D Sr. Anterior and nasal transposition of the inferior oblique muscles. J AAPOS 2003; 7: 167-173.

21 Ellis FJ. The pen, the pencil, and the inferior oblique. J AAPOS 2007; 11: 7-9.

22 Fard MA. Anterior and nasal transposition of the inferior oblique muscle for dissociated vertical deviation associated with inferior oblique muscle overaction. J AAPOS 2010; 14: 35-38.

23 Quinn AG, Kraft SP, Day C, Taylor RS, Levin AV. A prospective evaluation of anterior transposition of the inferior oblique muscle, with and without resection, in the treatment of dissociated vertical deviation. J AAPOS 2000; 4: 348-353. 